\title{
Training therapists to work with people with intellectual disability in Improving Access to Psychological Therapies (IAPT) services.
}

Dave Dagnan ${ }^{1}$

John Masson ${ }^{1}$

Richard Thwaites ${ }^{2}$

Amy James ${ }^{1}$

Chris Hatton ${ }^{3}$

${ }^{1}$ Cumbria Partnership NHS Foundation Trust

Community Learning Disability Services

Unit 1

Lakeland Business Centre

Jubilee Road

Workington

CA14 4HA

${ }^{2}$ First Step

Elmwood

2a Tynefield Drive

Penrith

Cumbria

CA11 8JA

${ }^{3}$ Centre for Disability Research

Division of Health Research

Faculty of Health and Medicine

Lancaster University

Lancaster

LA1 4YG

Address for Correspondence: Professor Dave Dagnan, Consultant Clinical Psychologist, Cumbria Partnership NHS Foundation Trust, Community Learning Disability Services, Unit 1, Lakeland Business Centre, Jubilee Road, Workington, CA14 4HA, Tel: 01900 705825, Fax: 01900 608127, Mobile 07789 868259, email: dave.dagnan@cumbria.nhs.uk

Key words: intellectual disability; psychological therapy; training; IAPT; confidence 


\section{Abstract}

Background: Current policy in the UK suggests that people with intellectual disabilities should, where possible, access mainstream mental health services. One factor that will enable good quality care is training for mainstream staff to support them in working with people with intellectual disabilities.

Method: Sixty-eight therapists working in an English Improving Access to Psychological Therapies (IAPT) received one or two day training on working with people with intellectual disabilities.

Measures of confidence, general therapeutic self-efficacy and attitudes to people with intellectual disabilities use of mainstream mental health services were completed pre-training, post-training and at three months follow-up; at which time 12 participants were interviewed to determine the impact of the training on their practice.

Results: There was a significant positive change in all measures immediately post-training which was maintained at 3 months follow-up.

Conclusions: Training considerations for mainstream therapists who may work with people with intellectual disabilities are discussed.

\section{Introduction}

People with intellectual disabilities experience higher rates of mental ill-health compared to those without intellectual disabilities (Cooper, Smiley, Finlayson et al., 2007) with a $6.6 \%$ prevalence for all affective disorders, 3.8\% for depression and 3.8\% for anxiety disorders (Cooper, Smiley, Morrison, Williamson, \& Allan, 2007). English policy suggests that people with intellectual disabilities and mental ill-health should receive mainstream services where appropriate (Department of Health, 2011). This is consistent with the Equality Act (Great Britain, 2010; Wadham, Ruebain, Robinson, \& Uppal, 2010) which puts a responsibility on public sector bodies to make 'reasonable adjustments' to make them accessible to protected groups of people including those with intellectual disabilities. Public sector bodies such as the National Health Service (NHS) have a duty to monitor their procedures to make sure their treatments for people with intellectual disabilities are accessible. The Monitor Compliance Framework standard 'Access to Health Care for People with a Learning Disability' requires English National Health Service Foundation Trusts to make reasonable adjustments to mainstream services to make them accessible to people with intellectual disabilities (Monitor, 2015). 
The policy demands in this area are clear, however, there is still relatively little literature describing models and outcomes that enable good quality care in mainstream mental health services for people with intellectual disabilities. Hall, Parkes, Samuels, \& Hassiotis (2006) described the evaluation of a service that promoted access to mainstream mental health services for people with intellectual disabilities. People with intellectual disabilities who received services in mainstream community and inpatient settings were assessed at three time points and were found to have similar mental health presentations to those without intellectual disabilities; however people with intellectual disabilities in mainstream inpatient settings had more unmet need and presented greater risk than those in community settings. People with intellectual disabilities in both community and inpatient settings showed significant improvements in the outcomes measured in the study. Donner, Mutter and Scior (2010) reported the views of service users, carers and providers regarding the experience of people with intellectual disabilities in mainstream inpatient mental health services. Whilst there were some positive aspects of this experience, the overall conclusion was that services lacked flexibility in meeting the needs of people with intellectual disabilities. Most of the research examining the experience of people with intellectual disabilities in mainstream settings is concerned with inpatient settings and there is no work of this type examining experience of mainstream psychological therapy.

There are a number of factors that may facilitate people with intellectual disabilities' use of mainstream mental health services. One such factor is the specialist knowledge and confidence of practitioners in working with this group (Rose, O'Brien, \& Rose, 2007). Werner and Stawski (2012) reviewed research examining the training needs of staff working with people with intellectual disabilities and mental ill-health. Studies reviewed by Werner and Stawski (2012) involved both professionals who worked with people with intellectual disabilities who were offered training in mental health issues and professionals who worked in mental health who were offered training in working with people with intellectual disabilities. The training programmes reviewed had a wide range of duration and content. Hemm, Dagnan and Meyer (2014) reviewed studies of training needs for health professionals who did not primarily work with people with intellectual disabilities. They found that training needs tended to be identified in the areas of general communication, role specific knowledge/information and profession and role specific needs.

Most of the research examining the experience of people with intellectual disabilities in mainstream settings is concerned with inpatient settings and most clinicians working in mainstream mental health services report little or no specific training on working with people with intellectual 
disabilities (Werner, Stawski, Polakiewicz, \& Levav, 2014), however, there are few accounts of training for mainstream talking therapists in working with people with intellectual disabilities. Dagnan, Masson, Cavagin, Thwaites, and Hatton (2015) describe the development of the Therapy Confidence Scale for therapists working with people with intellectual disabilities (TCS-ID). As part of the development of the scale they describe its use with four groups of therapists, three primary care therapy services (totalling 50 people) and one secondary care service (10 people). The data reported in that paper are only pre and post-training and are presented primarily to show the sensitivity of the scale to change, however they demonstrate a significant increase in therapist confidence in working with people with intellectual disabilities following training.

Primary care interventions for people with common mental health problems are substantially delivered through IAPT services, which are a continued UK government priority, and will be a major component of mental health pathways within developing Payment by Results structures. IAPT in England is a stepped care model; stepped care has two principles:

1. Treatment should always have the best chance of delivering positive outcomes while burdening the patient as little as possible.

2. A system of scheduled review to detect and act on non-improvement must be in place to enable stepping up to more intensive treatments, stepping down where a less intensive treatment becomes appropriate and stepping out when an alternative treatment or no treatment become appropriate.

Care in IAPT is delivered by two types of psychological therapy practitioners:

1. High Intensity Therapists trained in cognitive behavioural therapy for people with moderate and severe depression and anxiety disorders, and a range of other therapies as recommended by NICE. High Intensity practitioners are trained to British Association of Behavioural and Cognitive Psychotherapy accreditation standards (Clark, 2011).

2. Psychological Wellbeing Practitioners trained in cognitive behavioral approaches for people with mild to moderate anxiety and depression (Richards, 2010). These approaches include guided self-help and delivering psycho-educational groups. Services may also have administrative staff, employment advisors, a GP advisor and links with other services such as housing, drugs advice 
and benefits. The training curriculum for Primary Wellbeing Practitioners is highly structured and contains many methods that can be adapted for people with intellectual disabilities.

In 2013-2014 947,640 people were referred to IAPT services in England (Health and Social Care Information Centre, 2014), and IAPT Positive Practice Guidance has been published to support their access (Foundation for People with Learning Disabilities, 2015). The scale of IAPT resource available for the treatment of anxiety and depression in England is considerable, it is important that people with intellectual disabilities are able to access this where possible.

This paper presents an evaluation of a training curriculum designed to prepare High Intensity Therapists and Psychological Wellbeing Practitioners to work with people with intellectual disabilities.

\section{Method}

\section{Development of training curriculum}

The training curriculum reported here was based on the extensive experience of the project group of researching and providing cognitive therapy for people with intellectual disabilities and providing training to therapists in this area (e.g. Dagnan \& Chadwick, 1997; Dagnan, Jahoda, \& Kilbane, 2013; Dagnan, Kroese, \& Jahoda, 2007). The curriculum is a modularised program of eight sessions, each of around 75 minutes. The modularisation means that a core curriculum of two (sessions 1 and a combined session from session 3 and 5) or four sessions (Sessions 1, 3, 5 and 8) can be delivered as an introductory session or as a core session with a further four sessions (session 2, 4, 6 and 7) that build more depth in the core areas of adaptation of assessment and intervention that form the full the full eight sessions.

The eight sessions in the full curriculum are:

1. Introduction to intellectual disability, epidemiology, nature and causes. Impact of literacy and numeracy difficulties in the general population. This session emphasises the importance of adaptations to therapy to people with low IQ scores and not just those with clear diagnoses of intellectual disability. 
2. Stigma and its impact on therapy relationships. This session identifies the impact of intellectual disability on access to mental health services and on the formation of therapeutic alliance.

3. Introduction to the assessment of people with intellectual disabilities. This session considers the use of structured assessments with people with intellectual disabilities, and in particular focusses on the use of the GAD-7 (Spitzer, Kroenke, Williams, \& Lowe, 2006) and the PHQ-9 (Lowe, Kroenke, Herzog, \& Grofe, 2004) as these are the core measures used in IAPT services in England (Clark, 2011).

4. Advanced assessment of people with intellectual disabilities. This session discusses the assessment of core abilities of people with intellectual disability associated with successful engagement in CBT (Dagnan et al., 2007).

5. Overview of adaptation of therapeutic techniques. This session identifies the interventions from the therapist's core skill set that are easiest and hardest to use with people with intellectual disabilities.

6. Specific examples of adaptation; for example, thought diaries and behavioural activation. This session gives a detailed overview of adaptation for specific interventions. The interventions may vary in this session to meet the needs of the training group.

7. Therapeutic approaches and formulation. This session considers the underlying theoretical distinctions between therapies that are deficit and distortion based (Dagnan \& Chadwick, 1997) and considers the implications of this for assessment, formulation and intervention.

8. Overview of local services and discussion of communication and support systems. This session gives a detailed overview of local services and identifies pathways and supports available to mainstream therapists who might need support or supervision in working with people with intellectual disabilities.

Participants

A total of 32 Primary Wellbeing Practitioners received the four module training; six men and 26 women who had a mean age of 31.5 (SD = 8.2, range $25-55$ years) years. Twelve (37.5\%) had received some previous training on working with people with intellectual disabilities, 10 (31.3\%) had worked with people with intellectual disabilities in educational or residential settings and 15 (46.1\%) had worked therapeutically with people with intellectual disabilities. Two practitioners (6.3\%) considered themselves to work behaviourally, 31 (96.9\%) to work cognitive behaviourally, one (3.15\%) considered themselves eclectic. The group were asked to report how many of their current caseloads were people with intellectual disabilities (mean $=0.62, S D=0.89$; range $0-2$ ), how many 
were people with 'lower ability' (mean $=2.04, \mathrm{SD}=3.02$; range $0-10$ ) and how many were people with literacy problems (mean 2.59, SD = 3.55; range 0-15).

A total of 36 High Intensity Practitioners received the 8 module training; consisting of six men and 30 women who had a mean age of $44.9(S D=7.7)$ years, with an age range of 27-57 years. Twenty (55.6\%) had received some previous training on working with people with intellectual disabilities, nine $(25.0 \%)$ had worked with people with intellectual disabilities in educational or residential settings and 17 (47.2\%) had worked therapeutically with people with intellectual disabilities. Four practitioners $(11.1 \%)$ considered themselves to work behaviourally, and $36(100 \%)$ to work cognitive behaviourally. The group were asked to report how many of their current caseloads were people with intellectual disabilities (mean $=0.29, \mathrm{SD}=0.58$; range $0-2$ ), how many were people with 'lower ability' (mean $=1.71, S D=1.57$; range $0-5)$ and how many were people with literacy problems (mean $1.90, \mathrm{SD}=1.70$; range $0-6)$.

Measures

The following measure was completed at pre-training only

1. A demographic sheet which identified the name of the participant, their age, gender and job title. The sheet also requested information on previous training experienced, identified main therapeutic approach, experience of providing therapy for people with intellectual disabilities and the number of people with intellectual disabilities, lower ability and literacy/numeracy difficulties that the therapist believed they had on their caseload at the time of the training..

The following measures were all completed by participants pre-training, immediately post training and at 3 months follow-up.

2. The Therapy Confidence Scale - Intellectual Disabilities; (TCS-ID; Dagnan et al., 2015). This is a 14 item scale that describes the confidence of therapists in working with people with intellectual disabilities. The items are reproduced in Dagnan et al. (2015) and describe stages in engaging people in therapy, from forming a therapeutic alliance, carrying out assessments, formulating, adapting interventions and enabling a positive end to therapy. The TCS-ID has a single factor structure accounting for $62 \%$ of the variance, Cronbach's Alpha for the scale is 0.93 , test-retest 
reliability is 0.83 and the scale has been shown to be sensitive to change following training (Dagnan et al., 2015). Based on 64 people who completed the scale at pre-training in this study Cronbach's Alpha for the scale was 0.94; the data in the present study do not overlap with the data reported in Dagnan et al (2015).

3. A 5 item general therapy self-efficacy scale (Dagnan et al., 2015) using 5 items adapted from the General Self-Efficacy Scale (GSE; (Schwarzer \& Jerusalem, 1995)) where the general statement of problem was adapted to represent a general clinical problem. The scale was used to provide a measure of general clinical efficacy against which to compare the specific intellectual disability therapy confidence scale. Dagnan et al. (2015) reported properties of the scale from 107 mainstream therapists, the adapted scale has a Cronbach's Alpha of 0.69 and an adjusted itemtotal correlation range of $0.31-0.51$. Based on 64 people who completed the scale at pre-training in this study Cronbach's Alpha for the scale in this study was 0.75 .

4. Attitudes to the treatment of people with intellectual disabilities in mainstream services (adapted from Melville et al, 2005). These five items were not originally reported as a single scale but have a common theme regarding the perceived needs, perceived service priority, and perceived roles for IAPT and specialist intellectual disability staff in relation to people with intellectual disabilities and mental ill-health. Cronbach's Alpha for these 5 items based on 66 people who completed the scale at pre-training was 0.69 .

The following measure was completed at 3 months follow-up only

5. A qualitative interview was carried out with six Psychological Wellbeing Practitioners and six High Intensity Practitioners at three months post training. The interview lasted around 20 minutes and the topic guide first asked practitioners to reflect upon the training, then focussed on the extent to which participants had been able to use the skills acquired in the training and asked them to describe case studies of clients where the skills had helped their therapeutic work. The interviews were recorded and transcribed for analysis.

Procedure.

The participants in the training days were asked to complete the baseline questionnaires at the start of the first day, after an explanation of the importance of evaluating training interventions and 
assurance that if participants did not want to complete forms they would still complete the training. Around 15-20 minutes was allowed for completion of the questionnaires. The same procedure was followed at the end of the training intervention, with time allowed for completion of the questionnaires to ensure that the training days did not run over their allotted time. The three month follow-up data was collected by email with the follow-up questionnaire being emailed to all IAPT staff at 3 months. Two follow-up reminders were sent out at weekly intervals to all staff with a reassurance to those who had already returned the questionnaire.

Six High Intensity Therapists and six Psychological Wellbeing Practitioners were randomly selected from the group who had attended the training days. These people were emailed and asked if they were willing to be interviewed regarding the training course. If they were willing a time was set for the interview and the interview was recorded and transcribed. If they did not respond to the email, a further sample was taken from the initial training group until six people were interviewed from each group.

\section{Results}

Table 1 shows the scores for all scales at each time point. A between group comparison for age, gender, pre-training confidence scale scores, pre-training general efficacy scores and pre-training attitude scores was carried out to compare those people who completed scales at all time points with those who did not. No comparisons were statistically significant suggesting the group completing scales at all time points was representative of the full group. Table 1 shows scores only for the population that completed all scales at all time points. Shapiro Wilk's tests showed that confidence, efficacy and attitude scores were normally distributed at all time points for both groups; the data were therefore analysed using repeated measures Analysis of Variance.

A repeated-measures between-group analysis for the TCS-ID was carried out. Mauchly's test of sphericity was not significant ( $W=0.88, d f=2, p=0.09$ ) so no adjustments were needed. The main effect of time was significant $(F=55.1, d f=2, p<0.001$, partial eta squared $=0.57$ ) and the main effect for group was also significant $(F=23.0, d f=1, p<0.001$, partial eta squared $=0.35)$. There was no significant effect for the interaction of group and time $(F=0.35, d f=2, p=0.56$, partial eta squared $=0.01$ ). Although there is an effect for group, there is no interaction effect therefore post hoc repeated measures t-tests were carried out on the data combined across groups. The comparison between pre-training and post training was significant (mean difference $=11.3, \mathrm{SD}=6.5$, 
$t=11.2, d f=35, p<0.001)$, the comparison between post-training and follow-up scores for confidence was not significant (mean difference $=-1.7, \mathrm{SD}=7.6, \mathrm{t}=-1.5, \mathrm{df}=35, \mathrm{p}=0.14$ ), the comparison between pre-training and follow up was significant (mean difference $=9.4, S D=8.9, t=$ $7.0, d f=35, p<0.001)$.

A repeated measures between group analysis for the efficacy scale was carried out. Mauchly's test of sphericity was not significant ( $W=0.97, d f=2, p=0.19$ ) so no adjustments were needed. The main effect of time was significant $(F=25.7, d f=2, p<0.001$, partial eta squared $=0.39)$ and the main effect for group was also significant $(F=10.4, d f=1, p<0.005$, partial eta squared $=0.21)$. There was no significant effect for the interaction of group and time $(F=2.3, d f=2, p=0.11$, partial eta squared $=0.05)$. Although there is an effect for group, there is no interaction effect therefore post-hoc repeated measures t-tests were carried out on the data set combined across groups. The comparison between pre-training and post training was significant (mean difference $=1.5, \mathrm{SD}=1.9, \mathrm{t}$ $=4.8, \mathrm{df}=38, \mathrm{p}<0.001)$, the comparison between post-training and follow-up scores for confidence were not significant (mean difference $=-0.51, \mathrm{SD}=2.2, \mathrm{t}=-1.5, \mathrm{df}=38, \mathrm{p}=0.15$ ), the comparison between pre-training and follow up was significant (mean difference $=2.0, S D=1.7, t=7.0, d f=38$, $p<0.001)$.

A repeated measures between group analysis for the attitude scale was carried out. Mauchly's test of sphericity was not significant ( $W=0.96, d f=2, p=0.46$ ) so no adjustments were needed. The main effect of time was significant $(F=6.4, d f=2, p<0.05$, partial eta squared $=0.14)$ and the main effect for group was not significant $(F=0.4, d f=1, p=0.54$, partial eta squared $=0.01)$. There was no significant effect for the interaction of group and time $(F=0.05, d f=2, p=0.97$, partial eta squared 0.01). Post-hoc repeated measures t-tests were carried out on the data set combined across groups. The comparison between pre-training and post training was significant (mean difference $=1.1, \mathrm{SD}=$ 1.9, $t=3.8, d f=39, p<0.001)$, the comparison between post-training and follow-up scores for confidence were not significant (mean difference $=0.29, S D=2.0, t=-0.5, d f=39, p=0.58$ ), the comparison between pre-training and follow up was significant (mean difference $=1.0, S D=2.2, t=$ $2.8, d f=39, p<0.01)$.

\section{Qualitative analysis}

In order to add to the understanding of the effect of training and to gain insight from therapists' reflections on the impact of training on their clinical practice, six Primary Wellbeing practitioners and 
six High Intensity Practitioners were interviewed at three months follow-up; the interview was recorded and transcribed. The interview was analysed using thematic analysis (Guest, MacQueen, \& Namey, 2012). The transcription was used to create a data table with themes identified for each exchange. Themes were then collated and main themes were identified (fourth author). The full data table for all participants was shared with a second rater (first author) who agreed content against themes and validated themes. Any changes were discussed and consensus reached. There was generally good agreement in themes and exchanges allocated to themes. Themes identified were: 'increased awareness and sensitivity', 'adaptation and simplification of materials', 'adaptation and simplification of communications', and 'adapting interventions'.

Increased awareness and sensitivity

Seven respondents made reference to general increased awareness of the needs of people with intellectual disability and lower ability and identified broadly how that had influenced their practice.

I am more aware of it now, as before, I would maybe, think like, the questionnaire we fill in with the people, things like the PHQ and GAD, before I would give them to people and get them to fill them in and bring them back or give them to people and fill them in session, last time because I think I'm now more aware that people may struggle with that, I always now say to people,' do you want to fill that in or do you want to go through it together', and give them the option rather than embarrassing them by shoving it onto them.

\section{Adaptation and simplification of materials}

Ten respondents discussed the adaptation of materials.

....an understanding of how materials might be adapted for someone with a low cognitive ability that's kind of lower verbal understanding that kind of thing, better ability to think abstractly because CBT is quite often an abstract thing to either teach or talk to someone, yeah I found that helpful.

The focus in the training was on the principles of how to make adaptations; for example High Intensity Practitioners in session six were tasked with considering how thought diaries could be made more accessible. During the shared discussion exemplars were offered. However, a number of 
respondents reflected that they would have preferred more direction towards recommended materials with discussion of how to use them.

\section{Adaptation and simplification of communication.}

Eight respondents discussed adaptation of communication. Examples were given of using recordings of key passages in therapy for the client to take away from the session and of ensuring a shared language around key issues of emotion and cognition. Overall respondents identified an increased awareness of the need to adjust communication to the needs of the individual that they attributed to the training.

I've got one gentleman who said to me he can read stuff but his writing is very.... he doesn't have any confidence in writing and feels that this is an area that isn't good at all, so I have just had to go with his way of doing things so explaining more and checking for his understanding through conversation rather than checking he's written something down and see his understanding that way.

\section{Adaptation and simplification of interventions}

Ten respondents identified examples of changes in practice that reflected an increased use of principles in adaptation of intervention that recognised the need for simplification and repetition of interventions.

I can remember particularly working with one client.... we used cognitive restructuring and he said himself that he particularly found he struggled with thinking about things ... so we took a problem solving approach which was much more concrete and involved thinking out different steps and planning different steps which ultimately got the same results which I would of got to with cognitive restructuring in a way that he can grasp more because it was more involving and thinking about what he was actually doing than meta-cognitively.

Another therapist discussed their simplification of therapy for a client

.... I could easily start to talk about feelings and thoughts using abstract examples, but I didn't do that, I talked about his reactions to things and was able to get in that way and then 
explain to him that sometimes emotions feel stronger than others and again I was able to use his examples to talk about when it was worse, when it was better, when it was happening, that's kind of where it went. If I was working with someone without the lower ability it would be a bit more abstract and then maybe I would talk quicker as well, with him it was very much about his emotions and how they affected him and gave him an idea about you know what sort of influence that can have upon us

Discussion

This paper has described the outcomes of training for Improving Access to Psychological Therapies staff in working therapeutically with people with intellectual disabilities. Psychological Wellbeing Practitioners and High Intensity Therapists took part in a 1 or 2 day training course. Participants completed measures of confidence, attitude to working with people with intellectual disabilities in mainstream services and a general therapy efficacy measure pre, post and three months after training. Six members of each group were interviewed three months after training with a focus on their experience of using knowledge and skills gained in the training. Both groups reported significant positive changes in confidence, attitudes to working with people with intellectual disabilities in mainstream services and a general therapy efficacy immediately post training, which were maintained at 3 months post training.

Qualitative data showed that all participants interviewed could identify changes to their practice that they attributed to the training sessions. The four key themes from the interviews of, 'increased awareness and sensitivity', 'adaptation and simplification of communication', 'adaptation and simplification of materials' and 'adaptation and simplification of interventions' are consistent both with previously identified adaptations to therapy used with people with intellectual disabilities (Whitehouse, Tudway, Look, \& Kroese, 2006) and with training needs identified for mainstream staff considering working with people with intellectual disabilities (Hemm et al, 2015; Werner \& Stawski, 2012). Qualitative interviews were included in this study to give insight into the changes in 'skills' introduced by the training. Rose et al (2012) suggest that many training studies for staff working with people with intellectual disabilities have focussed on outcomes such as knowledge, and attitudes but often have not measured 'skills'. The qualitative approach adopted here demonstrates a 'competency' based approach with case examples of how the training had changed staff practice. 
The primary outcome for this training was confidence in working therapeutically with people with intellectual disabilities. Betz et al (2003) define confidence as 'a self-perceived ability to accomplish tasks', a definition similar to that of self-efficacy; this is an important concept as it suggests therapists may feel enabled to use core skills they already possess. Confidence increased over baseline immediately after training and remained significantly greater at three-month follow-up. Although the training curriculum offers new knowledge and skills for therapists in working with people with intellectual disabilities, the primary aim of the training is to enable minimal adaptation to meet the needs of the client. A consistent message within the training is that IAPT therapists have core skills in adaptation (indeed they often report that they adapt for every client) and that they already have the core skills required to meet the needs of people with mild and moderate intellectual disabilities. It is of interest that as specific confidence increases and remains significantly above baseline, the general therapy self-efficacy measure scores also increase for both groups. A tentative interpretation of this may be that the opportunity to reflect on the skills that therapists currently have and a structured experience of adaptation has increased therapists' general sense of their efficacy as therapists. A significant and consistent increase in positive attitude to people with intellectual disabilities in mainstream services is also evident, however, the measure used in this study was not designed as a single measure (Melville et al., 2005) and has a relatively low Cronbach's alpha. This is an area of measurement that might usefully be developed as a key outcome variable in training with mainstream services.

It is notable that there is no interaction of group and training for any outcome, which suggests that the changes are broadly equal for the group that received a single day and the group that received two days of training. It is also notable that there was a main effect for group in both the confidence and general efficacy measures. The Psychological Well-being Practitioners (PWPs) scored more highly on both scales throughout the intervention; an effect which was also noted in the paper describing the psychometric properties of the TCS-ID (Dagnan et al., 2015). The demographics of PWPs and the structured nature of the intervention they are trained to deliver may, in part, explain these effects. However these differences make a judgement regarding the relative effectiveness of one and two day training difficult and it may be worthwhile to compare training of different duration within groups with the same training and experience.

IAPT is commissioned to work with people with intellectual disabilities and Positive Practice guidance for IAPT services on working with people with intellectual disabilities has been published (Foundation for People with Learning Disabilities, 2015). The guidance identifies a number of areas 
that can act as barriers to people with intellectual disabilities using IAPT services. These include the referral and access processes and the skills of the therapists, However they also identify the relatively high literacy and numeracy requirements of the access and clinical processes used by IAPT services. For example IAPT protocols include session by session measurement using the Generalised Anxiety Disorder Assessment (GAD 7; (Spitzer et al., 2006) and the Patient Health Questionnaire-9 (PHQ-9; (Lowe et al., 2004). Both of these measures are brief and, in general, relatively simple however neither have been standardised for people with intellectual disabilities.

It is only through ensuring that systems and processes are accessible and that 'mainstream' therapists are confident in working with people with intellectual disability and lower ability that this client group will begin to get equitable access to therapy services. On this basis we would suggest that training for therapists to work with individuals of lower ability should be embedded within mainstream CBT courses to ensure that 'mainstream' therapists are equipped to assess and adapt therapies for this client group. The CBT competencies framework developed by Roth and Pilling (2007) identifies a number of 'CBT specific meta-competences' which involve the ability to adapt therapy in ways that will enable therapists to meet the needs of groups such as those with intellectual disabilities. A systematic approach to pre and post-qualification training in the area will support increased access to IAPT services by people with intellectual disabilities and will help therapists adapt their approaches for people with lower ability with similar needs to people with intellectual disabilities.

\section{Key Practitioner Message:}

- Policy in England suggests that, where possible, people with intellectual disabilities should be enabled to use mainstream mental health services.

- There have been no previous studies reporting training of mainstream psychological therapists to work with people with intellectual disabilities.

- Training for mainstream therapists has a positive impact of confidence in working with people with intellectual disabilities, general therapeutic self-efficacy and positive attitudes to people with intellectual disabilities using mainstream services. 
Table 1: Confidence, Self-Efficacy and Attitude scales pre-training, post-training and three month follow-up.

\begin{tabular}{|c|c|c|c|c|c|c|c|c|c|c|}
\hline & \multicolumn{4}{|c|}{ Pre } & \multicolumn{3}{|c|}{ Post } & \multicolumn{3}{|c|}{3 Month Follow-Up } \\
\hline & $\mathrm{N}$ & Mean & SD & Range & Mean & SD & Range & Mean & SD & Range \\
\hline Confidence scale PWP & 22 & 29.8 & 8.6 & $15-44$ & 41.4 & 7.0 & $29-53$ & 40.1 & 8.0 & $23-55$ \\
\hline Confidence scale $\mathrm{HI}$ & 22 & 21.3 & 8.7 & $5-36$ & 32.1 & 6.4 & $23-47$ & 29.9 & 10.2 & $14-47$ \\
\hline Self-efficacy scale PWP & 22 & 13.4 & 2.1 & $9-19$ & 14.2 & 2.7 & $10-19$ & 15.2 & 2.2 & $12-20$ \\
\hline Self-efficacy scale HI & 20 & 10.6 & 2.5 & $6-13$ & 12.7 & 2.8 & $6-15$ & 13.0 & 2.1 & $9-16$ \\
\hline Attitude scale PWP & 22 & 8.3 & 2.0 & $4-11$ & 9.4 & 1.7 & $6-13$ & 9.2 & 2.2 & $7-15$ \\
\hline Attitude scale $\mathrm{HI}$ & 20 & 8.1 & 2.0 & $3-13$ & 9.1 & 1.8 & $6-12$ & 8.9 & 1.6 & $6-13$ \\
\hline
\end{tabular}




\section{$\underline{\text { References }}$}

Betz, N. E., Borgen, F. H., Rottinghaus, P., Paulsen, A., Halper, C. R., \& Harmon, L. W. (2003). The expanded skills confidence inventory: Measuring basic dimensions of vocational activity. Journal of Vocational Behavior, 62, 76-100.

Clark, D. M. (2011). Implementing NICE guidelines for the psychological treatment of depression and anxiety disorders: the IAPT experience. International Review of Psychiatry, 23, 318-327.

Cooper, S. A., Smiley, E., Finlayson, J., Jackson, A., Allan, L., Williamson, A., et al. (2007). The prevalence, incidence, and factors predictive of mental ill-health in adults with profound intellectual disabilities. Journal of Applied Research in Intellectual Disabilities, 20, 493-501.

Cooper, S. A., Smiley, E., Morrison, J., Williamson, A., \& Allan, L. (2007). An epidemiological investigation of affective disorders with a population-based cohort of 1023 adults with intellectual disabilities. Psychological Medicine, 37, 873-882.

Dagnan, D., \& Chadwick, P. (1997). Cognitive behaviour therapy for people with learning disabilities: assessment and intervention. In B. S. Kroese, D. Dagnan \& K. Loumides (Eds.), Cogntive Behaviour Therapy for People with Learning Disabilities (pp. 110-123). London: Routledge.

Dagnan, D., Jahoda, A. J., \& Kilbane, A. (2013). Preparing People with Intellectual Disabilities for Psychological Treatment. In J. L. Taylor, W. R. Lindsay, R. P. Hastings \& C. Hatton (Eds.), Psychological Therapies for Adults with Intellectual Disabilities (pp. 55-68). Chichester: Wiley-Blackwell.

Dagnan, D., Kroese, B., \& Jahoda, A. (2007). Cognitive Behavioural Therapy and People with Intellectual Disabilities. In G. O'Reilly, J. McEvoy \& P. Walsh (Eds.), Handbook of Clinical Psychology and Intellectual Disability Practice. London: Routledge.

Dagnan, D., Masson, J., Cavagin, A., Thwaites, R., \& Hatton, C. (2015), The Development of a Measure of Confidence in Delivering Therapy to People with Intellectual Disabilities. Clinical Psychology and Psychotherapy, 22, 392-398

Department of Health. (2011). No Health Without Mental Health: a cross-government mental health outcomes strategy for people of all ages. Retrieved from https://www.gov.uk/government/uploads/system/uploads/attachment_data/file/213761/d h_124058.pdf.

Donner, B., Mutter, R., \& Scior, K. (2010). Mainstream In-Patient Mental Health Care for People with Intellectual Disabilities: Service User, Carer and Provider Experiences. Journal of Applied Research in Intellectual Disabilities, 23, 214-225.

Great Britain. (2010). The Equality Act. Retrieved from http://www.legislation.gov.uk/ukpga/2010/15/pdfs/ukpga_20100015_en.pdf. 
Guest, G., MacQueen, K. M., \& Namey, E. E. (2012). Applied Thematic Analysis. California: Sage.

Hall, I., Parkes, C., Samuels, S., \& Hassiotis, A. (2006). Working across boundaries: clinical outcomes for an integrated mental health service for people with intellectual disabilities. Journal of Intellectual Disability Research, 50, 598-607.

Health and Social Care Information Centre. (2014). Psychological Therapies, Annual Report on the use of IAPT services,. Retrieved from http://www.hscic.gov.uk/catalogue/PUB14899/psycther-ann-rep-2013-14.pdf

Hemm, C., Dagnan, D. and Meyer, T. D. (2015), Identifying Training Needs for Mainstream Healthcare Professionals, to Prepare Them for Working with Individuals with Intellectual Disabilities: A Systematic Review. Journal of Applied Research in Intellectual Disabilities, 28, 98-110.

Foundation for People with Learning Disabilities (2015). Learning Disabilities Positive Practice Guide. Retrieved from http://www.mentalhealth.org.uk/publications/FPLD-positive-practiceguide/.

Lowe, B., Kroenke, K., Herzog, W., \& Grofe, K. (2004). Measuring depression outcome with a brief self-report instrument: sensitivity to change of the Patient Health Questionnaire (PHQ-9). Journal of Affective Disorders, 81, 61-66.

Melville, C. A., Finlayson, J., Cooper, S. A., Allan, L., Robinson, N., Burns, E., et al. (2005). Enhancing primary health care services for adults with intellectual disabilities. Journal of Intellectual Disability Research, 49, 190-198.

Monitor (2015) Risk Assessment Framework 2013/14. Retrieved from https://www.gov.uk/government/uploads/system/uploads/attachment_data/file/455893/R AF_revised_25_August.pdf.

Richards, D. A. (2010). Reach Out: National Programme Supervisor Materials to Support the Delivery of Training for Psychological Wellbeing Practitioners Delivering Low Intensity Interventions: Rethink.

Rose, N., O'Brien, A., \& Rose, J. (2007). Investigating staff knowledge and attitudes towards working with adults with learning disabilities and mental health difficulties. Advances in Mental Health and Learning Disabilities, 1, 52-59.

Rose, N., Rose, J., \& Kent, S. (2012). Staff training in intellectual disability services: a review of the literature and implications for mental health services provided to individuals with intellectual disability. International Journal of Developmental Disabilities, 58, 24-39.

Roth, A. D., \& Pilling, S. (2007). The competencies required to deliver effective cognitive and behavioural therapy for people with depression and with anxiety disorders: Improving Access 
to Psychological Therapies (IAPT) Programme. Retrieved from

https://www.ucl.ac.uk/pals/research/cehp/research-

groups/core/pdfs/cbt/Backround_CBT_document_-_Clinicians_version.pdf

Schwarzer, R., \& Jerusalem, M. (1995). Generalized Self-Efficacy scale. In J. Weinman, S. Wright, \& M. Johnston (Eds) Measures in health psychology: A user's portfolio. Causal and control beliefs (pp. 35-37). Windsor, UK: NFER-NELSON.

Spitzer, R. L., Kroenke, K., Williams, J. B. W., \& Lowe, B. (2006). A brief measure for assessing generalized anxiety disorder - The GAD-7. Archives of Internal Medicine, 166, 1092-1097.

Wadham, J., Ruebain, D., Robinson, A., \& Uppal, S. (2010). Blackstone's Guide to the Equality Act 2010: OUP Oxford.

Werner, S., \& Stawski, M. (2012). Mental health: Knowledge, attitudes and training of professionals on dual diagnosis of intellectual disability and psychiatric disorder. Journal of Intellectual Disability Research, 56, 291-304.

Werner, S., Stawski, M., Polakiewicz, Y., \& Levav, I. (2014). Psychiatrists' knowledge, training and attitudes regarding the care of individuals with intellectual disability. Journal of Intellectual Disability Research, 57, 774-782.

Whitehouse, R. M., Tudway, J. A., Look, R., \& Kroese, B. S. (2006). Adapting individual psychotherapy for adults with intellectual disabilities: A comparative review of the cognitive-behavioural and psychodynamic literature. Journal of Applied Research in Intellectual Disabilities, 19, 5565. 